\title{
In-situ Atomic-Scale Visualization of Atomic-Step Induced NiO growth during the Oxidation of $\mathrm{Ni}$
}

\author{
Xiaobo Chen ${ }^{1}$, Jianyu Wang ${ }^{2}$, Xianhu Sun ${ }^{3}$, Dmitri Zakharov ${ }^{4}$, Sooyeon Hwang ${ }^{5}$ and Guangwen Zhou ${ }^{3}$ \\ ${ }^{1}$ Binghamton University, Port Jefferson Station, New York, United States, ${ }^{2}$ Binghamton University, New \\ York, United States, ${ }^{3}$ Binghamton University, United States, ${ }^{4}$ Brookhaven National Lab, United States, \\ ${ }^{5}$ Brookhaven National Lab, New York, United States
}

The interaction of reactive gases with metallic surfaces is crucial to many technological applications, ranging from corrosion problems to heterogenous catalysis. Significant attention has been focused on elucidating the effect of structural and compositional inhomogeneities of metallic surfaces in the active state (i.e., clean surfaces) on oxygen adsorption and the resulting oxide nucleation and growth during the initial stages of oxidation $[1,2]$. The atomic-level and microscopic mechanisms underlying the interactions of oxygen with a metal surface in the passive state (i.e., covered with an oxide layer) still remain largely unexplored. This is because the insulating (or wide-gap) nature of bulk oxides prohibits the use of many surface-sensitive techniques that are based on the detection of charged particles (e.g., electrons and ions) and cannot be applied to studying the oxide surfaces.

In contrast, transmission electron microscopy (TEM) is not subject to such a limitation and is capable of providing atomic-scale information for both the surface and subsurface regions of the oxide. Particularly, environmental transmission electron microscopy (ETEM) offers unique opportunities to study the bulk oxide growth at the atomic scale with the temperature-, pressure-, and time-resolved imaging of the surface oxidation [3]. Herein, we use ETEM to atomically resolve the surface growth of a thick NiO layer during the oxidation of $\mathrm{Ni}$ and identify the lively dynamics of surface steps in controlling the oxide growth. Ni foils with a nominal thickness of $\sim 50 \mathrm{~nm}$ were prepared by using the focus ion beam (FIB) lift-out technique. The Ni foils were subsequently annealed at $\sim 450{ }^{\circ} \mathrm{C}$ under hydrogen gas flow inside the ETEM column to remove the native oxide and produce tears and holes in the Ni foil. The facets along these freshly created holes are oxide-free and ideal for in situ visualization of the oxidation process. In situ TEM observation of the oxide growth was made in cross-sectional views by imaging the side facets of the oxide formed along the edges of the empty holes in the Ni foil.

Figs. 1(a-c) illustrate in situ high-resolution TEM (HRTEM) images, along the [110] zone axis, of the $\mathrm{NiO}$ growth during the oxidation of $\mathrm{Ni}$ at $\mathrm{pO}_{2}=1.2 \times 10^{-4}$ Torr and $450{ }^{\circ} \mathrm{C}$. As marked by the dashed cyan line along the $\mathrm{NiO} / \mathrm{Ni}$ interface in Fig. 1(a), the $\mathrm{NiO}$ and the $\mathrm{Ni}$ substrate can be distinguished by the interplanar spacing of the crystal lattice $(2.1 \AA$ for $\mathrm{NiO}(100)$ vs. $2.0 \AA$ for $\mathrm{Ni}(111))$. Figs. 1(b, c) display a monoatomic step of $\mathrm{NiO}$ propagating laterally via step flow toward the right. The step flow of the oxide layer indicates that the oxide growth occurs via an adatom process by surface diffusion of $\mathrm{Ni}$ and $\mathrm{O}$ atoms, where $\mathrm{Ni}$ atoms are supplied by the outward diffusion of $\mathrm{Ni}$ from the $\mathrm{Ni}$ substrate through the $\mathrm{NiO}$ layer to the $\mathrm{NiO}$ surface and $\mathrm{O}$ atoms are supplied from the surrounding $\mathrm{O}_{2}$ atmosphere. Figs. 1(d-f) depict in situ HRTEM image snapshots showing the lateral flow of multiple atomic steps and resulting bunching of the steps because of the difference in the flow speed of the atomic steps. As marked by arrows 1-3 in Fig. 1(d), the NiO surface has three monoatomic steps. Steps 1 and 2 move toward the right and step 3 propagates in the opposite direction. Step 1 moves at a faster rate and catches up with step 2, thereby resulting in the bunching of the two steps into a double-layer step. The bunched step moves toward the right at a much slower rate than the monoatomic steps (i.e., steps 1 and 3) (Fig. 1(e, f)). Figs. 1(g-i) present in situ HRTEM images showing the oxide growth induced debunching of the multiple-layer steps. As illustrated in Fig. 3(g), the NiO surface consists of triple-layer steps. The bottom layer of the bunched step grows at a faster rate, resulting in the formation of a monoatomic step and a bi-layer step. This bottom monoatomic step propagates toward the left and reaches the edge of the $\mathrm{NiO}$ 
island (Figs. 1(h-i)). During the same time period, the double-layer and triple-layer steps on the right side remain relatively stationary, indicating that the oxide growth is dominated by the lateral flow of monoatomic steps. The in situ TEM visualization provides the microscopic details about the oxidation of Ni in the passive state. Atomic modelling will be performed to correlate the in-situ HRTEM imaging and elucidate the oxide growth mechanism, mainly focusing on the effects of surface steps on the surface diffusion of reactants.
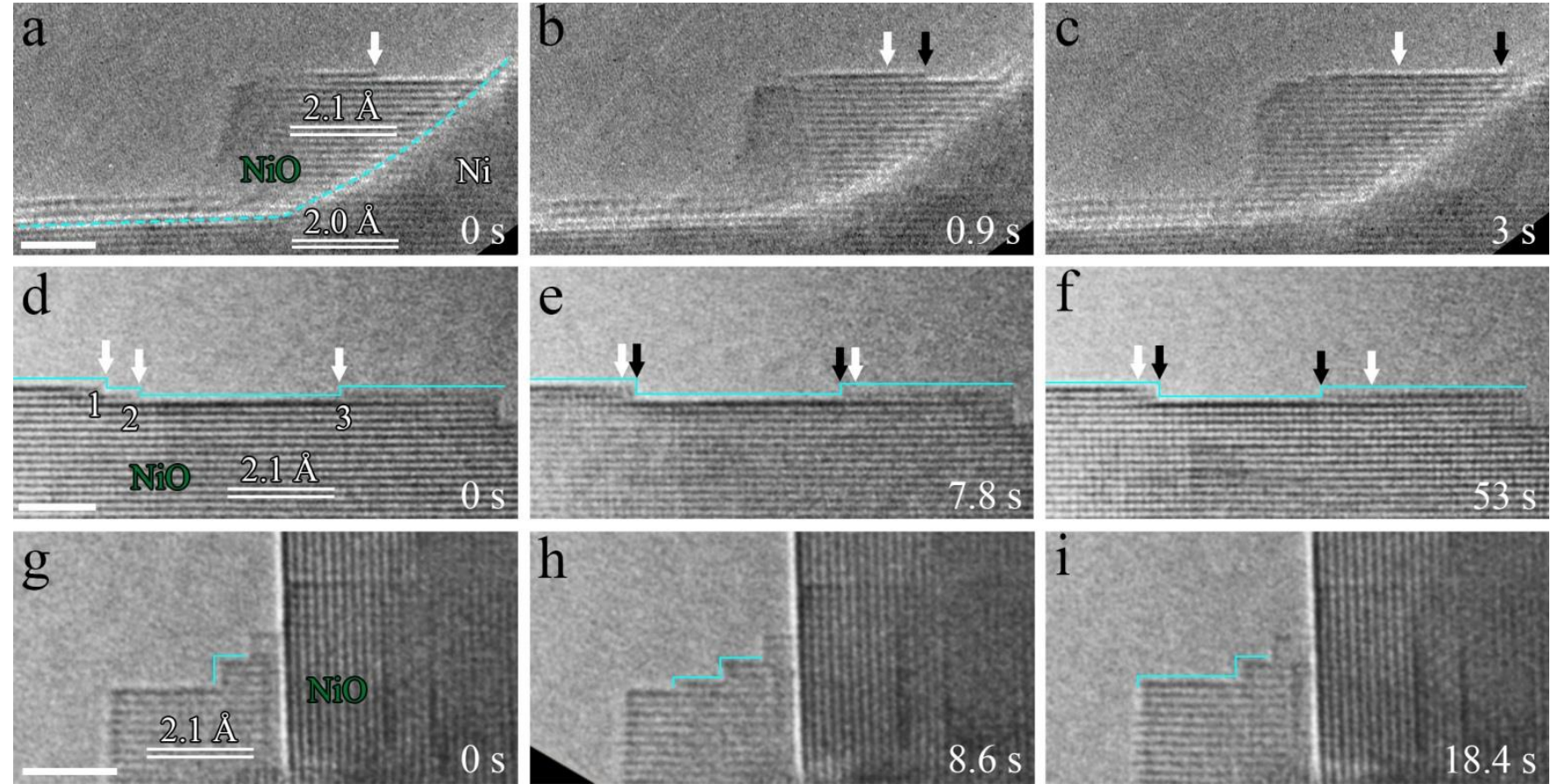

Figure 1. In situ HRTEM imaging of the oxidation of $\mathrm{Ni}$ at $450{ }^{\circ} \mathrm{C}$ and $\mathrm{pO} 2=1.2 \times 10-4$ Torr. (a-c) In situ HRTEM images illustrating the lateral propagation of a monoatomic step of $\mathrm{NiO}$. The dashed cyan line marks the oxide/metal interface in (a). (d-f) Time-sequenced HRTEM images displaying the bunching of two monoatomic steps via the faster growth of the upper step. The black arrows mark the oxide growth by the lateral propagation of the step and white arrows mark the location of the monoatomic step at $0 \mathrm{~s}$ and serve as a reference point used for measuring the propagation speed of the monoatomic step in (a-c) and the bunched step in (d-f), respectively. (g-h) In situ HRTEM snapshots showing the debunching of a triple-layer step into a monolayer step and a double-layer step, via the faster growth of the bottom step. The solid cyan lines mark the triple-layer step and its subsequent debunching. Scale bar, $2 \mathrm{~nm}(\mathrm{a}-\mathrm{i})$.

\section{References}

[1] Qin, H., et. al., Proc. Natl. Acad. Sci. U.S.A. 112, E103 (2015)

[2] Li, L., et. al., Phys. Rev. Lett. 113, 136104 (2014)

[3] Sharma, R., Microsc. Microanal. 7, 494; (2001)

[4] This work was supported by the U.S. Department of Energy, Office of Basic Energy Sciences, Division of Materials Sciences and Engineering under Award No. DE-SC0001135. This research used resources of the Center for Functional Nanomaterials, which is a U.S. DOE Office of Science Facility, at Brookhaven National Laboratory under Contract No. DE-SC0012704. 